\title{
Caracterização da pecuária bovina no Estado da Paraíba, Nordeste do Brasil
}

\section{Characterization of cattle raising in Paraíba State, Northeastern Brazil}

\author{
Inácio José Clementino ${ }^{1}$; Carla Lauise Rodrigues Menezes Pimenta ${ }^{1}$; \\ Leise Gomes Fernandes ${ }^{1}$; Camila de Souza Bezerra ${ }^{2}$; Clebert José Alves ${ }^{3}$; \\ Ricardo Augusto Dias ${ }^{4}$; Marcos Amaku'; Fernando Ferreira \\ Evelise Oliveira Telles"; Vitor Salvador Picão Gonçalves ${ }^{5}$; \\ José Soares Ferreira Neto ${ }^{4}$; Sérgio Santos de Azevedo ${ }^{3 *}$
}

\section{Resumo}

Objetivou-se caracterizar a pecuária bovina no Estado da Paraíba, Nordeste do Brasil. O Estado da Paraíba foi dividido em três circuitos produtores de bovinos, levando-se em consideração as suas mesorregiões: circuito produtor 1 (mesorregião do Sertão), circuito produtor 2 (mesorregião da Borborema) e circuito produtor 3 (mesorregiões do Agreste e Zona da Mata). No total, foram selecionadas aleatoriamente 689 propriedades rurais distribuídas nos três circuitos produtores. As variáveis utilizadas na caracterização foram tipo de exploração, tipo de criação, tipo de ordenha, uso de inseminação artificial, resfriamento do leite, número de vacas em lactação, produção diária de leite, tamanho do rebanho e número de fêmeas bovinas com idade $>24$ meses em relação ao total de bovinos. Pelos resultados obtidos constatou-se que a maioria das explorações pecuárias paraibanas é familiar ou de subsistência, com predominância de exploração mista, criação semi-confinada, com utilização de ordenha manual e monta natural, sem emprego de resfriamento do leite produzido, com baixo número de vacas em lactação e baixa produtividade diária de leite. Foram encontradas diferenças e semelhanças entre os circuitos produtores, de maneira que se sugere que tais aspectos sejam levados em consideração quando do planejamento de políticas de desenvolvimento pecuário, bem como na implementação de ações de controle de doenças. Palavras-chave: Bovinocultura, sistemas de produção, características produtivas

\footnotetext{
Abstract

The aim of this work was to characterize the cattle raising in Paraíba State, Northeastern Brazil. The State was divided into three cattle production regions according to its mesoregions: production region 1 (Sertão mesoregion), production region 2 (Borborema mesoregion) and production region 3 (Agreste and Zona da Mata mesoregions). Overall, 689 rural properties from the three production regions were randomly selected. The variables used in the characterization were production system, farming

${ }^{1}$ Discentes do Curso de Doutorado em Medicina Veterinária, Universidade Federal de Campina Grande, UFCG, Patos, PB. E-mail: clementinoij@yahoo.com.br; carlalauise@hotmail.com; leisegf@hotmail.com

2 Discente do Curso de Graduação em Medicina Veterinária da UFCG, Patos, PB. E-mail: camila_cstr.mv@hotmail.com

3 Profs., UFCG, Patos, PB. E-mail: clebertja@uol.com.br; sergio.azevedo@pq.cnpq.br

${ }^{4}$ Profs., Universidade de São Paulo, USP, São Paulo, SP. E-mail: dias@vps.fmvz.usp.br; amaku@vps.fmvz.usp.br; fernando@ vps.fmvz.usp.br; bufalo@usp.br; jsoares@vps.fmvz.usp.br

${ }^{5}$ Prof., Universidade de Brasília, UnB, Brasília, DF. E-mail: vitorspg@unb.br

* Autor para correspondência
} 
system, type of milking, use of artificial insemination, milk cooling, number of lactating cows, daily milk production, herd size and number of bovine females $>24$ months of age. It was found that most farms in Paraíba State are family or subsistence farms, predominantly mixed production, semi-intensive farming, with utilization of hand milking and natural mating, without use of milk cooling, low number of lactating cows and daily milk production. It were found differences and similarities among production regions so that it is suggested that such aspects must be taking into account in livestock development public policy planning, as well as in the implementation of disease control strategies.

Key words: Cattle, production systems, production characteristics

\section{Introdução}

A bovinocultura é uma das principais atividades produtivas do agronegócio brasileiro, proporcionando a maior renda obtida entre as cinco maiores cadeias produtivas agropecuárias estudadas (CNA, 2012), sendo desenvolvida em todo o território nacional com 212,8 milhões de cabeças (IBGE, 2012), distribuídas em cerca de 2,67 milhões de estabelecimentos pecuários (IBGE, 2009). Com este efetivo bovino o Brasil possui o segundo maior rebanho mundial em número de cabeças, em número de abates e em produção de carne (USDA, 2011b), e desde 2008 ocupa o primeiro lugar em exportações de carne bovina (MAPA, 2013).

Em 2011 o Brasil ocupou a sexta posição mundial na produção de leite, entretanto, a quantidade de vacas em lactação foi proporcionalmente maior e alcançou a terceira posição (USDA, 2011a) devido à menor produtividade de leite do nosso rebanho. O rebanho bovino brasileiro está concentrado, principalmente, nas regiões Centro-Oeste, Norte e Sudeste, que somadas à região Sul possuem $86,1 \%$ do rebanho nacional. Estas regiões utilizam mais tecnologia na produção pecuária bovina, enquanto o Nordeste possui 13,9\% do rebanho (IBGE, 2012).

Os sistemas de produção e a tecnificação da pecuária bovina brasileira variam entre os estados. De acordo com o regime de alimentação, os sistemas de produção pecuária brasileira podem ser classificados em três tipos: extensivo, semiextensivo e intensivo (CEZAR et al., 2005). Para Miguel et al. (2007) os condicionantes para os diferentes sistemas de produção podem ser de ordem cultural, ecológica (determinantes do meio físico - solo e clima), ou até mesmo conjunturais (preços e mercados). Desse modo, no Brasil, observam-se diferentes sistemas de produção da bovinocultura e grandes diferenças nos níveis de produtividade de acordo com a organização do capital produtivo (POLAQUINI; SOUZA; GEBARA, 2006), além de objetivos ou finalidades da criação bastante diversificados, tanto entre as regiões geográficas quanto dentro de um mesmo estado, principalmente no Nordeste do Brasil.

Existem vários estudos sobre a caracterização da bovinocultura no Brasil (CARVALHO et al., 2001; ALMEIDA et al., 2006; CARNEIRO et al., 2006; SILVEIRA et al., 2006; MIGUEL et al., 2007; MOURA et al., 2010b; SILVA et al., 2010), no entanto, não há uma padronização na metodologia de avaliação e caracterização dos sistemas produtivos que se torna mais complicado pela elevada quantidade de características que precisam ser analisadas (PEREIRA, 2001).

A Paraíba possui 1,3 milhão de bovinos (IBGE, 2012) distribuídos em 92 mil estabelecimentos pecuários (IBGE, 2009), sendo a pecuária responsável por 2\% do PIB do Estado (IDEME, 2011), no entanto, não há estudos baseados em amostragem planejada e em nível estadual com o objetivo de caracterizar a pecuária bovina na Paraíba. O conhecimento das características e formas de produção pecuária de determinada região fornece subsídios importantes para planejamento das políticas de desenvolvimento pecuário, bem como para estudos epidemiológicos e programas de controle de doenças. Considerando estes aspectos foi estruturado o presente trabalho, que teve como objetivo caracterizar a pecuária bovina paraibana. 


\section{Material e Métodos}

A pesquisa foi realizada no Estado da Paraíba, ponto mais oriental das Américas, que ocupa uma área de 56.584,6 $\mathrm{km}^{2}$, sendo geograficamente dividida em quatro mesorregiões (Zona da Mata, Agreste, Borborema e Sertão), 23 microrregiões e 223 municípios (IBGE, 2001). A mesorregião do Sertão é tipicamente tradicional, caracterizandose por uma estrutura fundiária concentrada. Embora na mesorregião do Sertão predomine o clima semiárido, é na parte central do Estado, na mesorregião da Borborema, que se registram os menores índices pluviométricos. As mesorregiões do Agreste e Zona da Mata apresentam os maiores índices pluviométricos do Estado (IBGE, 2009).
O trabalho de campo foi realizado por Médicos Veterinários e técnicos do Serviço de Defesa Agropecuária do Estado da Paraíba, no período de setembro de 2012 a junho de 2013, e tal trabalho incluiu a visita às propriedades rurais e aplicação de questionário.

O Estado da Paraíba foi dividido em três circuitos produtores de bovinos, levando-se em consideração as suas mesorregiões (Figura 1): circuito produtor 1 (mesorregião do Sertão), circuito produtor 2 (mesorregião da Borborema) e circuito produtor 3 (mesorregiões do Agreste e Zona da Mata). Essa divisão também levou em conta a capacidade operacional e logística do serviço veterinário oficial do Estado para a realização das atividades de campo, baseando-se nas áreas de atuação de suas unidades regionais.

Figura 1. Mapa demonstrando a divisão do Estado da Paraíba em circuitos produtores de bovinos.

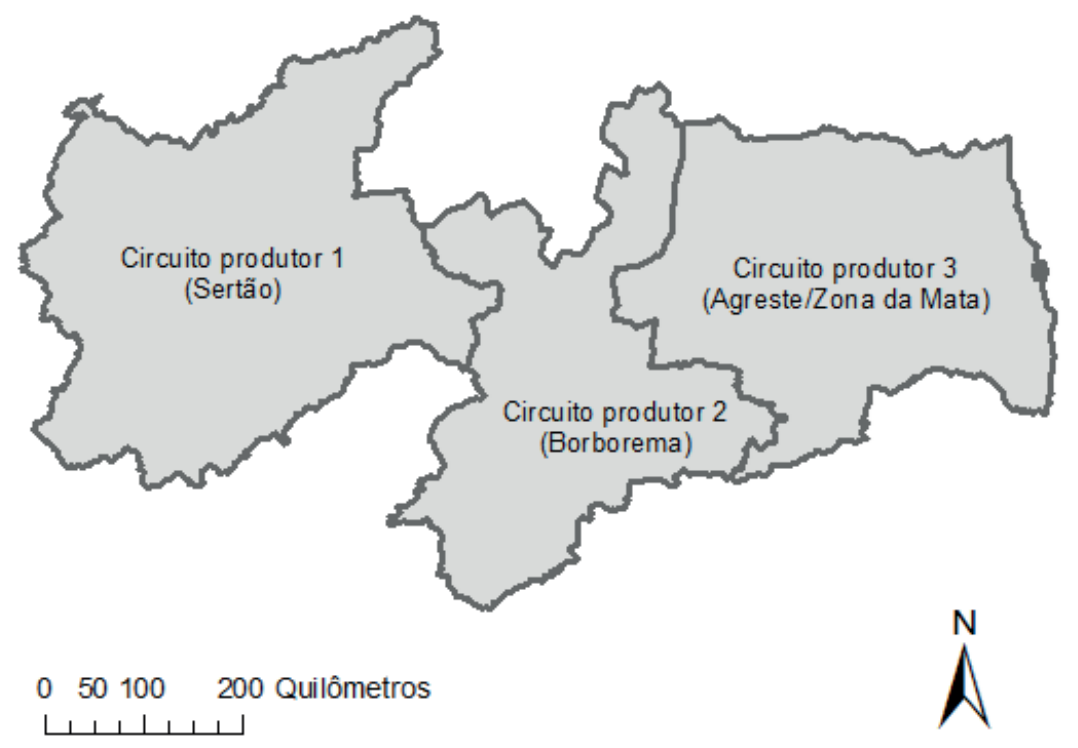

Fonte: Elaboração dos autores.

A amostragem das propriedades rurais circuito produtor foi estimado pela fórmula para foi realizada como parte do inquérito amostras simples aleatórias (THRUSFIELD, soroepidemiológico da brucelose bovina, no âmbito 1995). Os parâmetros adotados para o cálculo do Programa Nacional de Controle e Erradicação foram: nível de confiança de $95 \%$, prevalência de da Brucelose e Tuberculose Bovina (PNCEBT). brucelose bovina estimada em $25 \%$ e erro de $5 \%$. O número de propriedades selecionadas por A capacidade operacional e financeira do serviço 
veterinário oficial do Estado também foi levada em consideração para a determinação do tamanho da amostra por circuito. No total, foram selecionadas aleatoriamente e visitadas 689 propriedades rurais distribuídas nos três circuitos produtores.

Em cada propriedade visitada foi aplicado um questionário epidemiológico elaborado para obter informações que possibilitassem a caracterização da pecuária estadual. As variáveis qualitativas e respectivas categorias utilizadas foram tipo de exploração (corte, leite, mista), tipo de criação (intensiva, semi-intensiva, extensiva), tipo de ordenha (não ordenha, ordenha manual), inseminação artificial (não utiliza, utiliza só inseminação artificial) e resfriamento do leite (não, sim). As variáveis quantitativas utilizadas foram número de vacas em lactação, produção diária de leite, produtividade diária de leite (produção diária de leite dividida pelo número de vacas em lactação), tamanho do rebanho e número de fêmeas bovinas com idade $>24$ meses em relação ao total de bovinos.

Para as variáveis quantitativas, antes da escolha do teste estatístico a ser empregado para a comparação dos três circuitos produtores, primeiramente foi efetuado o teste de normalidade de Shapiro-Wilk, e para variáveis com distribuição normal foi utilizada análise de variância (ANOVA) com um critério de classificação utilizando-se o teste de Tukey para as comparações múltiplas; as variáveis sem distribuição normal foram analisadas pelo teste de Kruskal-Wallis e comparações múltiplas pelo teste de Dunn (ZAR, 1999). Para as variáveis qualitativas foram calculados a frequência e o intervalo de confiança de $95 \%$, e a comparação entre os circuitos foi realizada com o teste de qui-quadrado ou teste exato de Fisher. O nível de significância adotado foi de $5 \%$ e os cálculos foram efetuados com os programas SPSS 13.0 for Windows, EpiInfo versão 6.0 e BioEstat versão 5.03 .

\section{Resultados}

Os resultados da análise das variáveis qualitativas são apresentados nas Tabelas 1 a 5. Com relação ao tipo de exploração, houve predomínio das explorações pecuárias do tipo mista nos três circuitos pecuários e no âmbito estadual (Tabela 1). Houve diferença estatística das explorações de corte e mista entre os três circuitos produtores, e em relação ao tipo leite o circuito produtor 1 diferiu dos circuitos 2 e 3 .

Tabela 1. Frequência dos tipos de exploração em propriedades rurais do Estado da Paraíba segundo os circuitos produtores.

\begin{tabular}{ccccccccccc}
\hline \multirow{2}{*}{$\begin{array}{c}\text { Circuito } \\
\text { produtor }\end{array}$} & \multicolumn{9}{c}{ Corte } & \multicolumn{9}{c}{ Leite } & \multirow{2}{*}{ Total (\%) } \\
\cline { 2 - 10 } & N & \% & IC 95 \% & N & $\%$ & IC 95 \% & N & $\%$ & IC 95 \% & \\
\hline 1 & 10 & $4,4^{\mathrm{a}}$ & {$[2,1-7,9]$} & 43 & $18,9^{\mathrm{a}}$ & {$[14,1-24,7]$} & 174 & $76,7^{\mathrm{a}}$ & {$[70,6-82,0]$} & $227(100)$ \\
2 & 27 & $11,8^{\mathrm{b}}$ & {$[7,9-16,7]$} & 74 & $32,3^{\mathrm{b}}$ & {$[26,3-38,8]$} & 128 & $55,9^{\mathrm{b}}$ & {$[49,2-62,4]$} & $229(100)$ \\
3 & 50 & $21,8^{\mathrm{c}}$ & {$[16,7-27,8]$} & 80 & $34,9^{\mathrm{b}}$ & {$[28,8-41,5]$} & 99 & $43,2^{\mathrm{c}}$ & {$[36,7-49,9]$} & $229(100)$ \\
Estado & 87 & 12,7 & {$[10,3-15,4]$} & 197 & 28,8 & {$[25,4-32,3]$} & 401 & 58,5 & {$[54,7-62,3]$} & $685(100)$ \\
\hline
\end{tabular}

Na mesma coluna, letras sobrescritas minúsculas diferentes indicam diferença estatística $(\mathrm{p}<0,05)$ entre as frequências.

Fonte: Elaboração dos autores.

A descrição dos sistemas de produção segundo Houve predominância do sistema de criação semio tipo de criação está apresentada na Tabela 2. confinado no Estado e nos três circuitos produtores. 
Houve diferença estatística entre os circuitos 1 e 2 no tocante ao tipo de criação confinada, e entre os circuitos 1 e 2 e 2 e 3 com relação aos tipos semiconfinada e extensiva.

Tabela 2. Frequência dos tipos de criação em propriedades rurais do Estado da Paraíba segundo os circuitos produtores.

\begin{tabular}{ccccccccccc}
\hline \multirow{2}{*}{$\begin{array}{c}\text { Circuito } \\
\text { produtor }\end{array}$} & \multicolumn{9}{c}{ Tipo de criação } & \multirow{2}{*}{ Total (\%) } \\
\cline { 2 - 10 } & \multicolumn{1}{c}{ Confinada } & \multicolumn{1}{c}{ Semi-confinada } & IC 95 \% & N & $\%$ & IC 95 \% & N & $\%$ & IC 95 \% & \\
\hline 1 & 7 & $3,1^{\mathrm{a}}$ & {$[1,2-6,3]$} & 114 & $50,2^{\mathrm{a}}$ & {$[43,5-56,9]$} & 106 & $46,7^{\mathrm{a}}$ & {$[40,1-53,4]$} & $227(100)$ \\
2 & 20 & $8,7^{\mathrm{b}}$ & {$[5,4-13,1]$} & 169 & $73,5^{\mathrm{b}}$ & {$[67,3-79,1]$} & 41 & $17,8^{\mathrm{b}}$ & {$[13,1-23,4]$} & $230(100)$ \\
3 & 15 & $6,6^{\mathrm{a}} \mathrm{b}$ & {$[3,7-10,7]$} & 117 & $51,5^{\mathrm{a}}$ & {$[44,8-58,2]$} & 95 & $41,9^{\mathrm{a}}$ & {$[35,4-48,6]$} & $227(100)$ \\
Estado & 42 & 6,1 & {$[4,5-8,2]$} & 400 & 58,5 & {$[54,7-62,2]$} & 242 & 35,4 & {$[31,8-39,1]$} & $684(100)$ \\
\hline
\end{tabular}

Na mesma coluna, letras sobrescritas minúsculas diferentes indicam diferença estatística $(p<0,05)$ entre as frequências.

Fonte: Elaboração dos autores.

Com relação às variáveis relacionadas ao nível de tecnificação, na maioria das propriedades, nos três circuitos e no âmbito estadual, era empregada ordenha manual (sem ocorrência de propriedades com ordenha mecânica), não era utilizada inseminação artificial e não era feito resfriamento do leite (Tabelas 3, 4 e 5). Houve diferença estatística entre os circuitos 1 e 3 e 2 e 3 no relativo ao tipo de ordenha (Tabela 3), no entanto, não foi observada diferença estatística entre os circuitos com relação à utilização de inseminação artificial (Tabela 4) e resfriamento do leite (Tabela 5).

Tabela 3. Frequência do tipo de ordenha em propriedades rurais do Estado da Paraíba segundo os circuitos produtores.

\begin{tabular}{cccccccc}
\hline \multirow{3}{*}{ Circuito produtor } & \multicolumn{9}{c}{ Tipo de ordenha } & \multirow{2}{*}{ Total (\%) } \\
\cline { 2 - 6 } & \multicolumn{9}{c}{ Não ordenha } & IC 95\% & $\mathrm{N}$ & $\%$ & IC 95\% & \\
\cline { 2 - 6 } & $\mathrm{N}$ & \multicolumn{1}{c}{ Manual } & \\
\hline 1 & 29 & 13,3 & {$[9,1-18,5]$} & 189 & $86,7^{\mathrm{a}}$ & {$[81,5-90,9]$} & $21800)$ \\
2 & 32 & 15,9 & {$[11,2-21,7]$} & 169 & $84,1^{\mathrm{a}}$ & {$[78,3-88,8]$} & $201(100)$ \\
3 & 57 & 26,5 & {$[20,7-32,9]$} & 158 & $73,5^{\mathrm{b}}$ & {$[67,1-79,3]$} & $215(100)$ \\
Estado & 118 & 18,6 & {$[15,7-21,9]$} & 516 & 81,4 & {$[78,1-84,3]$} & $634(100)$ \\
\hline
\end{tabular}

Na mesma coluna, letras sobrescritas minúsculas diferentes indicam diferença estatística $(p<0,05)$ entre as frequências.

Fonte: Elaboração dos autores.

Tabela 4. Frequência da utilização de inseminação artificial em propriedades rurais do Estado da Paraíba segundo os circuitos produtores.

\begin{tabular}{cccccccc}
\hline \multirow{3}{*}{ Circuito produtor } & \multicolumn{9}{c}{ Utilização de inseminação artificial } & \multirow{3}{*}{ Total (\%) } \\
\cline { 2 - 6 } & \multicolumn{4}{c}{ Não utiliza } & \multicolumn{4}{c}{ Utiliza só inseminação artificial } & \\
\cline { 2 - 6 } & $\mathrm{N}$ & $\%$ & IC 95\% & $\mathrm{N}$ & $\%$ & IC 95\% & \\
\hline 1 & 218 & 100 & {$[98,3-100]$} & 0 & $0,0^{\mathrm{a}}$ & {$[0,0-1,7]$} & $218(100)$ \\
2 & 224 & 99,1 & {$[96,8-99,9]$} & 2 & $0,9^{\mathrm{a}}$ & {$[0,1-3,2]$} & $226(100)$ \\
3 & 217 & 99,1 & {$[96,7-99,9]$} & 2 & $0,9^{\mathrm{a}}$ & {$[0,1-3,3]$} & $219(100)$ \\
Estado & 659 & 99,4 & {$[98,5-99,8]$} & 4 & 0,6 & {$[0,2-1,5]$} & $663(100)$ \\
\hline
\end{tabular}

Na mesma coluna, letras sobrescritas minúsculas diferentes indicam diferença estatística $(p<0,05)$ entre as frequências.

Fonte: Elaboração dos autores. 
Tabela 5. Frequência do resfriamento do leite produzido em propriedades rurais do Estado da Paraíba segundo os circuitos produtores.

\begin{tabular}{cccccccc}
\hline \multirow{3}{*}{ Circuito produtor } & \multicolumn{9}{c}{ Resfriamento do leite } & \multirow{2}{*}{ Total (\%) } \\
\cline { 2 - 6 } & \multicolumn{9}{c}{ Não resfria } & \multicolumn{3}{c}{ Resfria } \\
\cline { 2 - 6 } & $\mathrm{N}$ & $\%$ & IC 95\% & $\mathrm{N}$ & $\%$ & IC 95\% & \\
\hline 1 & 218 & 98,2 & {$[95,5-99,5]$} & 4 & $1,8^{\mathrm{a}}$ & {$[0,5-4,5]$} & $222(100)$ \\
2 & 200 & 95,2 & {$[91,4-97,7]$} & 10 & $4,8^{\mathrm{a}}$ & {$[2,3-8,6]$} & $210(100)$ \\
3 & 205 & 97,6 & {$[94,5-99,2]$} & 5 & $2,4^{\mathrm{a}}$ & {$[0,8-5,5]$} & $210(100)$ \\
Estado & 623 & 97,0 & {$[95,4-98,2]$} & 19 & 3,0 & {$[1,8-4,6]$} & $642(100)$ \\
\hline
\end{tabular}

Na mesma coluna, letras sobrescritas minúsculas diferentes indicam diferença estatística $(p<0,05)$ entre as frequências.

Fonte: Elaboração dos autores.

Os resultados da análise das variáveis quantitativas são apresentados nas Figuras 2 a 6 . Com relação ao número de vacas em lactação, as medianas nos estratos amostrais são apresentadas na Figura 2, sendo o circuito produtor 1 estatisticamente diferente dos circuitos 2 e $3(\mathrm{p}<0,05)$. Para o Estado da Paraíba, o valor mediano foi de 3,0 vacas em lactação (primeiro quartil $=1,0$; terceiro quartil $=5,0$ ).

Figura 2. Número de vacas em lactação em propriedades rurais do Estado da Paraíba segundo os circuitos produtores.

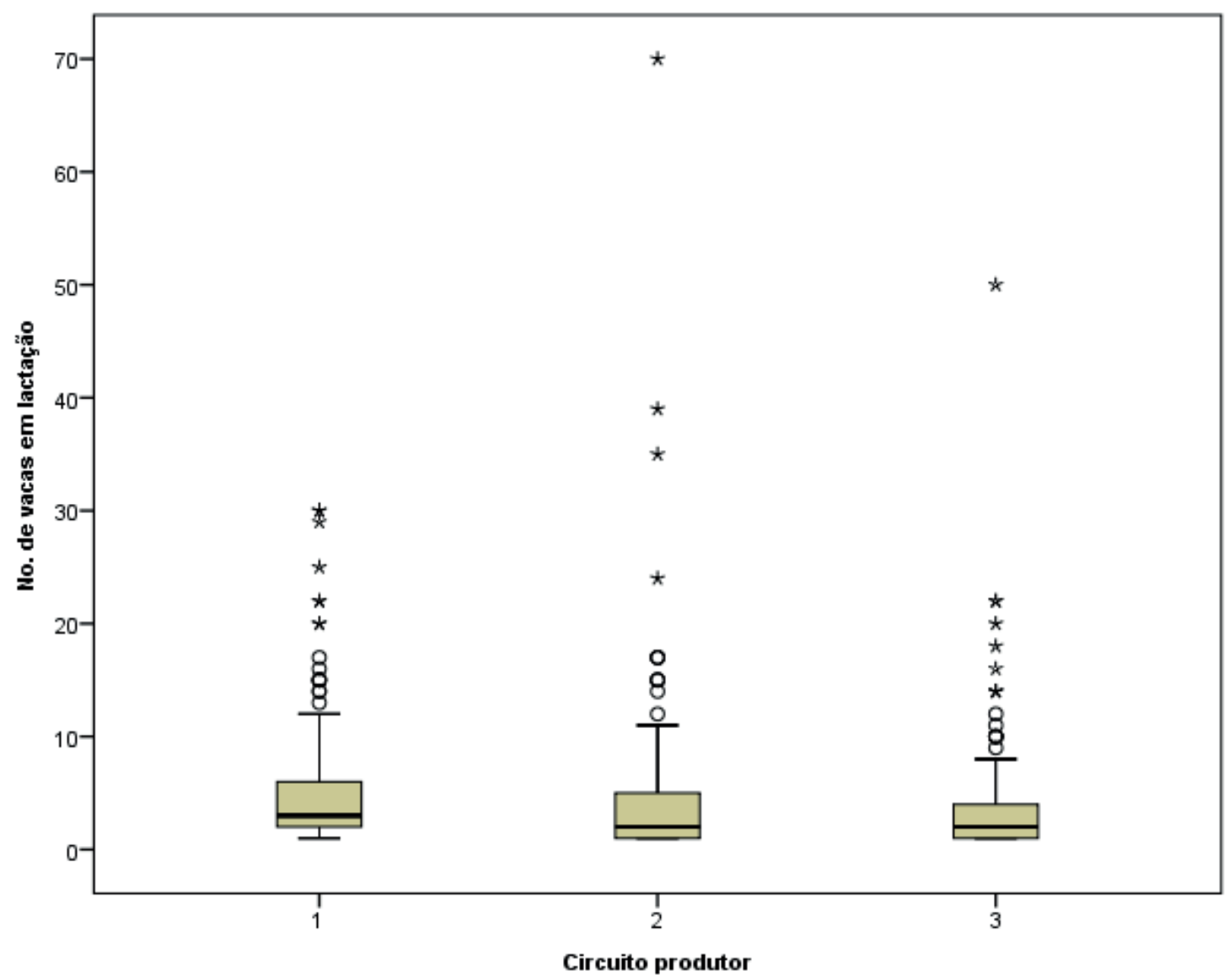

Fonte: Elaboração dos autores. 
As medianas da produção diária de leite nos circuitos produtores são apresentadas na Figura 3, não havendo diferença estatística entre os circuitos produtores $(p>0,05)$. No âmbito estadual, a mediana da produção de leite foi de 10,0 litros (primeiro quartil $=5,0$; terceiro quartil $=20,0)$. Com o número de vacas em lactação e a produção diária de leite, foi construída outra variável, a produtividade diária de leite (Figura 4). Com relação a essa variável, o circuito produtor 1 foi estatisticamente diferente dos circuitos 2 e $3(\mathrm{p}<0,05)$. Para o Estado, a mediana foi de 4,0 litros por vaca (primeiro quartil $=2,5$; terceiro quartil $=6,0)$.

Figura 3. Produção diária de leite (em litros) em propriedades rurais do Estado da Paraíba segundo os circuitos produtores. Paraíba, 2013.

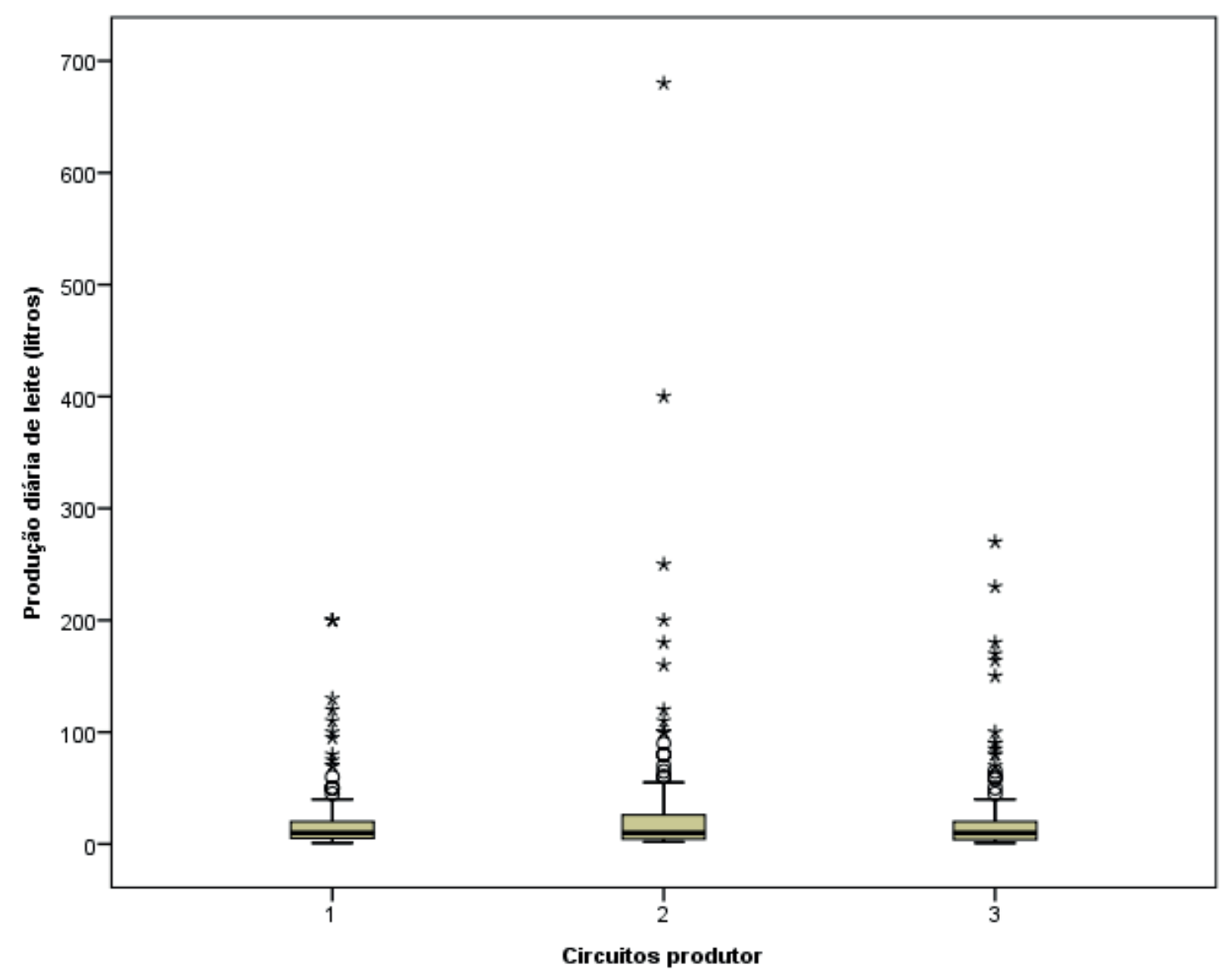

Fonte: Elaboração dos autores. 
Figura 4. Produtividade diária de leite (em litros) em propriedades rurais do Estado da Paraíba segundo os circuitos produtores. Paraíba, 2013.

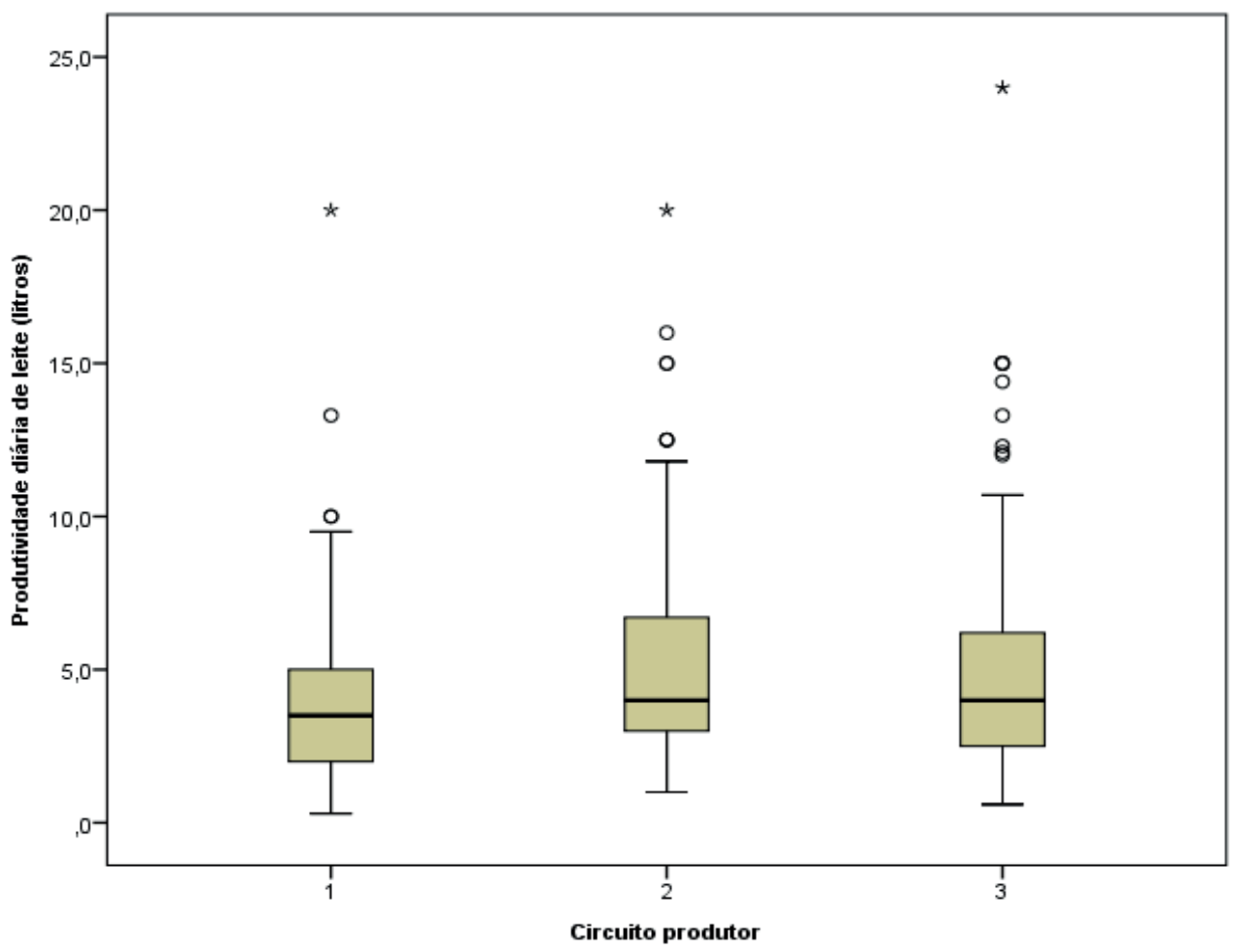

Fonte: Elaboração dos autores.

Figura 5. Número total de bovinos em propriedades rurais da Paraíba segundo os circuitos produtores. Paraíba, 2013.

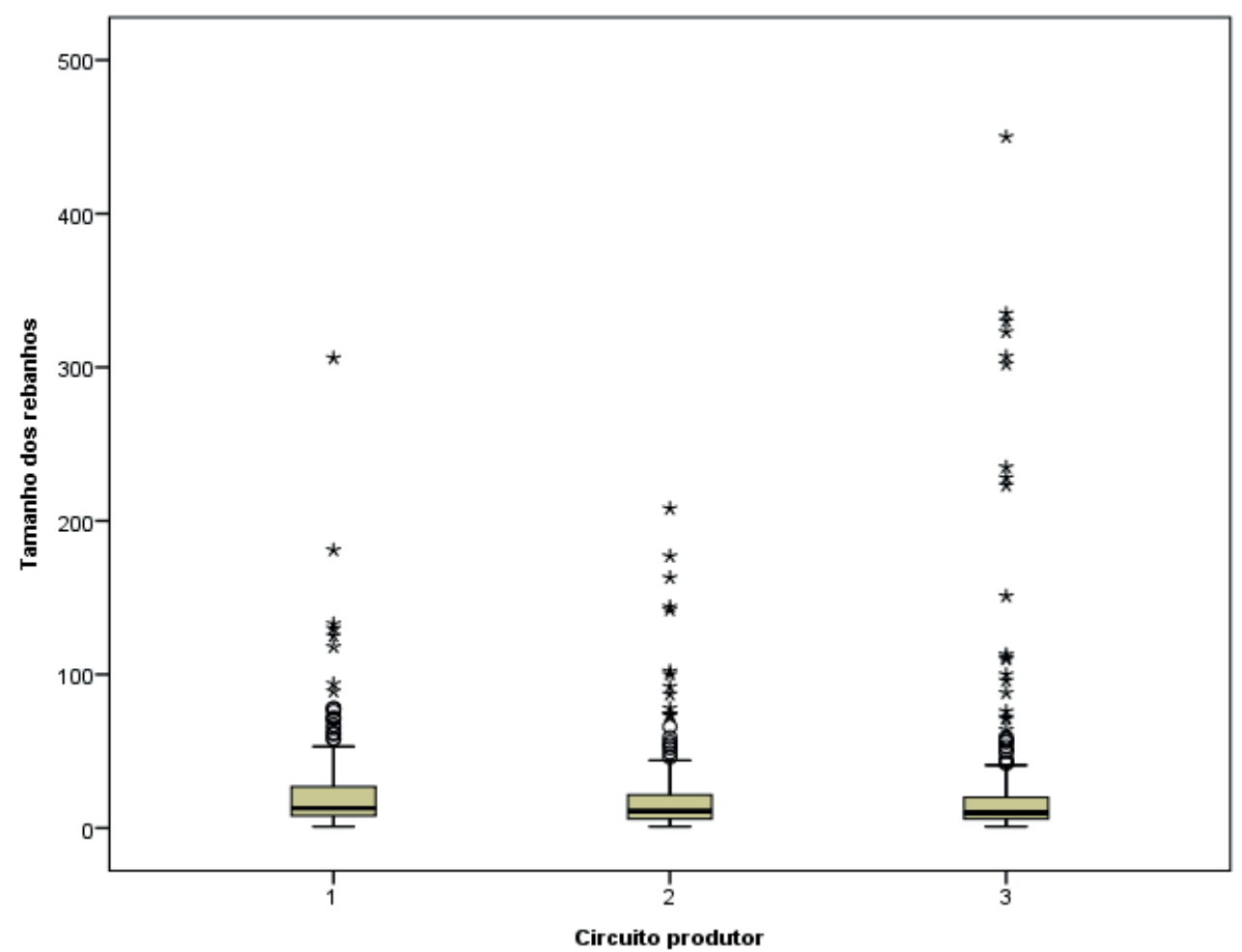

Fonte: Elaboração dos autores. 
Figura 6. Fêmeas bovinas maiores de 24 meses de idade em relação ao total de bovinos em propriedades rurais do Estado da Paraíba segundo os circuitos produtores. Paraíba, 2013.

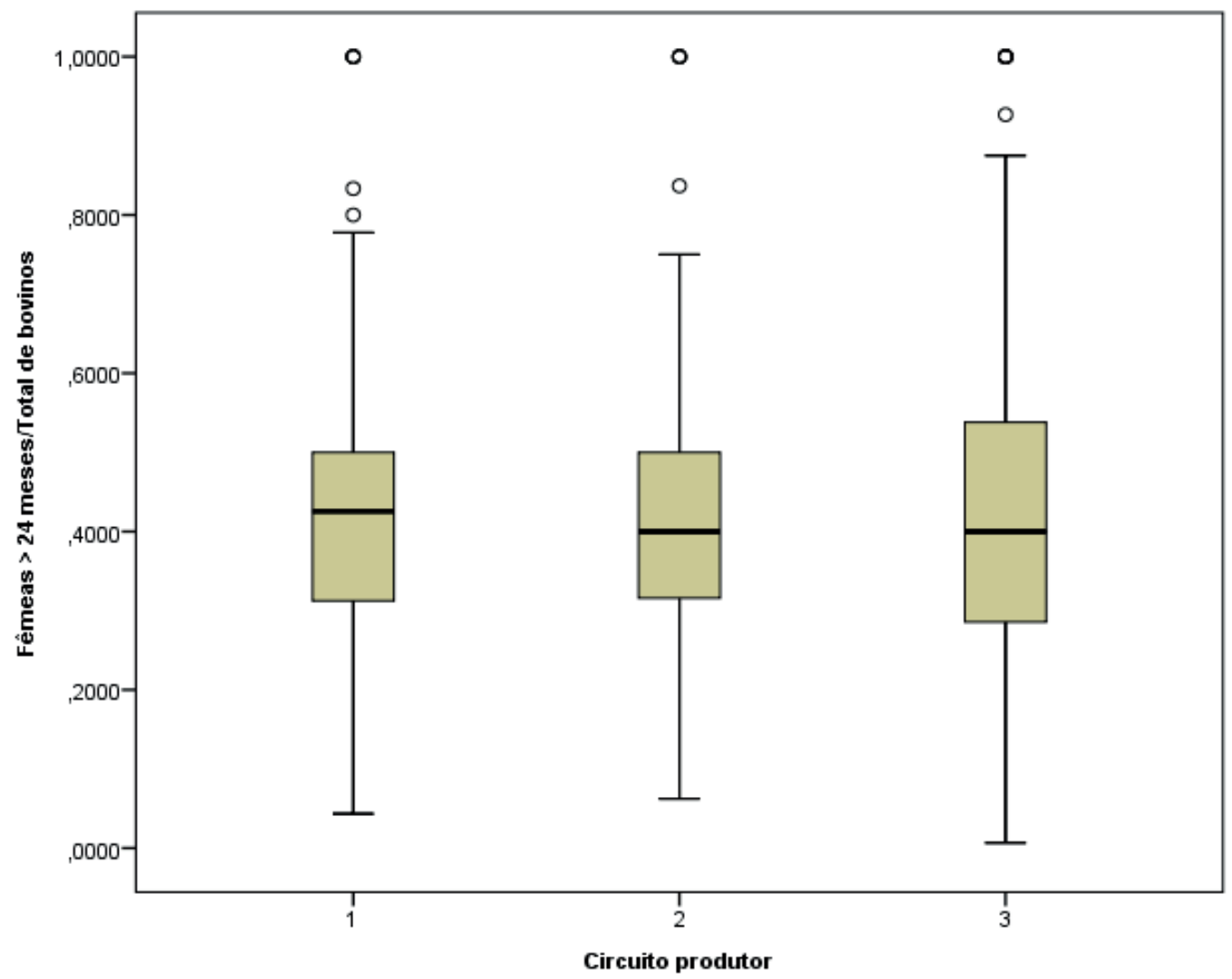

Fonte: Elaboração dos autores.

O número total de animais dos rebanhos (tamanho do rebanho) está apresentado na Figura 5. Nas propriedades amostradas, no âmbito estadual, o menor rebanho era composto por um animal e o maior por 450 animais, com mediana de 11,0 animais por rebanho (primeiro quartil $=7,0$; terceiro quartil $=23,0)$. O circuito produtor 1 foi estatisticamente diferente dos circuitos 2 e $3(p<0,05)$. No tocante à proporção de fêmeas bovinas com idade $>24$ meses em relação ao total de bovinos (Figura 6), não foi observada diferença estatística entre os circuitos produtores $(p>0,05)$. No Estado, a mediana foi de 0,40 fêmeas $>24$ meses/total de bovinos (primeiro quartil $=0,30$; terceiro quartil $=0,50$ ).

\section{Discussão e Conclusões}

A caracterização da amostra dos rebanhos bovinos do Estado da Paraíba foi realizada com os dados obtidos em questionário aplicado nas propriedades, e por meio desta caracterização foi possível a identificação da tipologia das mesmas e a verificação da ocorrência de possíveis semelhanças ou diferenças entre os três circuitos produtores de bovinos.

No Estado da Paraíba e nos circuitos produtores individualmente predominou o tipo de exploração mista, com diferenças entre os três circuitos produtores, destacando-se o circuito produtor 1, com 76,7\% das propriedades. Esses resultados corroboram os estudos realizados na Paraíba, Bahia, São Paulo e Santa Catarina, nos quais também houve predominância desse tipo de exploração (DIAS, 2004; LEITE et al., 2004; SIKUSAWA, 2004; ALVES, 2008), no entanto, vale salientar 
que no trabalho realizado anteriormente na Paraíba (LEITE et al., 2004), foram utilizadas apenas 72 propriedades rurais com efetivo bovino maior que 32 animais. No presente trabalho, as propriedades foram selecionadas com base em amostragem planejada, o que garante a inferência dos resultados obtidos.

Houve predominância do tipo de criação semiconfinado tanto no Estado quanto nos circuitos produtores, destacando-se o circuito produtor 2, com 73,5\% dos rebanhos criados de forma semiconfinada. Esses resultados diferem dos obtidos em estudos conduzidos com a mesma técnica amostral nos Estados de Santa Catarina, Rondônia, São Paulo, Maranhão e Bahia, nos quais predominou o sistema extensivo (DIAS, 2004; SIKUSAWA, 2004; ALVES, 2008; VILLAR, 2008; BORBA, 2012). Já nos Estados da Paraíba e Pernambuco, em rebanhos leiteiros, predominou o sistema semi-confinado ou confinado (CARNEIRO et al., 2006; MOURA et al., 2010a; NEVES et al., 2011). O sistema de confinamento apresentou a menor frequência no Estado e nos circuitos, sendo o circuito produtor 1 com a menor frequência $(3,1 \%)$, estatisticamente diferente dos outros dois circuitos produtores; por outro lado, os circuitos 2 e 3 apresentaram frequências estatisticamente semelhantes. Achados similares foram observados por Villar (2008), Sikusawa (2004), Dias (2004) e Borba (2012), nos Estados de Rondônia, Santa Catarina, São Paulo e Maranhão, respectivamente. O sistema de produção com animais confinados é frequentemente utilizado na pecuária de corte, situando-se, em sua maioria, nos Estados da região Centro-Oeste (CEZAR et al., 2005), no entanto, é o tipo de criação mais comum em algumas situações, como por exemplo em explorações especializadas em produção de leite e em áreas pequenas com pouca disponibilidade de forragem (CARNEIRO et al., 2006; NEVES et al., 2011).

A predominância do sistema semi-confinado encontrado neste estudo pode ser decorrente de características do Estado da Paraíba em relação ao quantitativo de animais, cuja média por exploração pecuária é de 24 bovinos. Devido à existência de pequenas explorações associada às condições climáticas do Estado, os produtores tem o hábito de prender os animais para fornecimento de ração no cocho. Uma hipótese que não pode ser descartada é a influência da seca que ocorreu no ano de 2012, o que levou os produtores a administrarem concentrado e volumoso no cocho em decorrência da escassez de pastagem.

Analisando-se as variáveis relacionadas ao nível de tecnificação (tipo de ordenha, utilização de inseminação artificial e resfriamento do leite) das propriedades, constatou-se que, no Estado da Paraíba, tal nível é baixo. No Estado e nos circuitos produtores houve predominância da ordenha manual, sem ocorrência de propriedades com ordenha mecânica. Dados semelhantes foram observados nos Estados de Santa Catarina e Espírito Santo (SIKUSAWA, 2004; AZEVEDO, 2006), no entanto, no Estado de São Paulo predominou a ordenha mecânica (DIAS, 2004). Já no Estado do Maranhão, Borba (2012) referiu que mais da metade dos produtores investigados não ordenhava suas vacas. Os dados encontrados no presente trabalho também coincidem com aqueles obtidos nos Estados de Pernambuco, Paraíba e Piauí, em que nas propriedades com pecuária leiteira estudadas predominou a ordenha manual (LEITE et al., 2004; CARNEIRO et al., 2006; MONTEIRO et al., 2007; NEVES et al., 2011).

$\mathrm{Na}$ Paraíba o manejo reprodutivo é feito predominantemente através da monta natural, no entanto, em $0,9 \%$ das propriedades dos circuitos produtores 2 e 3 a inseminação artificial é a forma de manejo reprodutivo utilizada. Em outros estudos, utilizando a mesma metodologia, constatou-se que houve predominância do uso da monta natural nos Estados de Rondônia, Santa Catarina, Espírito Santo, Maranhão, São Paulo e Bahia (DIAS, 2004; SIKUSAWA, 2004; AZEVEDO, 2006; ALVES, 2008; VILLAR, 2008; BORBA, 2012). No Estado da Paraíba, o uso da inseminação artificial limita-se 
a um número pequeno de criadores de raças mais especializadas. Segundo Carneiro et al. (2006), em trabalho sobre caracterização e eficiência de rebanhos leiteiros no Piauí, a eficiência reprodutiva dos rebanhos deve ser melhorada com a utilização de práticas de manejo mais adequadas, destacandose, nesse contexto, a utilização de inseminação artificial para melhoria genética do rebanho.

A maioria dos produtores paraibanos não faz o resfriamento do leite, coincidindo com os dados de estudos realizados nos Estados do Maranhão, São Paulo e Espírito Santo (DIAS, 2004; AZEVEDO, 2006; BORBA, 2012), no entanto, em algumas regiões específicas de produção leiteira, o resfriamento do leite é realizado frequentemente (SIKUSAWA, 2004), diferentemente dos achados de Carneiro et al. (2006), que constataram que na área leiteira de um programa de leite no Piauí, apenas $7,1 \%$ das propriedades possuía tanque de resfriamento.

Foi constatado que, no Estado da Paraíba, o menor rebanho era composto por um animal e o maior por 450 animais, com média de 24 e mediana de 11 animais por rebanho, constatando-se que o circuito produtor 1 foi estatisticamente diferente dos circuitos 2 e 3. Estes dados, juntamente com o conhecimento do número de fêmeas com idade superior a 24 meses, possibilitou o cálculo da proporção destas fêmeas em relação ao total de bovinos do rebanho, encontrando-se uma mediana estadual de 0,40, não havendo diferenças estatísticas entre os circuitos produtores. Com relação ao número de vacas em lactação foi observado, para o Estado da Paraíba, média de 4,6 e mediana de 3 vacas por exploração, sendo o circuito produtor 1 o que apresentou a maior média/mediana, diferindo estatisticamente dos circuitos 2 e 3 . Essas vacas foram responsáveis pela produção mediana diária de leite por exploração pecuária de 10 litros, sem diferença estatística entre os circuitos produtores. Desse modo, ao calcular-se a produtividade diária de leite foi encontrada mediana de 4 litros por vaca/ dia, sendo o circuito produtor 1 o que apresentou a menor mediana. Das 513 propriedades com vacas em lactação, a percentagem média de vacas em lactação em relação ao número total de vacas da propriedade foi de 62,5\%. Gomes (2005) e Moura et al. (2010b) sugeriram que para a exploração leiteira ser rentável, no mínimo, 75\% das vacas da criação devem estar em lactação, e que o aumento da competitividade dos sistemas de produção de leite depende do aumento da escala de produção.

Pelos resultados obtidos neste trabalho nota-se que a maioria das explorações pecuárias paraibanas é familiar ou de subsistência, com predominância de exploração mista, criação semi-confinada, com utilização de ordenha manual e monta natural, sem emprego de resfriamento do leite produzido, com baixo número de vacas em lactação e baixa produtividade diária de leite.

O conhecimento das características e formas de produção pecuária de determinada região fornece subsídios importantes para planejamento das políticas de desenvolvimento pecuário, bem como para estudos epidemiológicos e programas de controle de doenças. Os parâmetros epidemiológicos de determinadas doenças podem variar de acordo com certas características, como sistema de produção, tipo de manejo dos animais, tipo de exploração e tamanho médio dos rebanhos. Foi constatado que os circuitos produtores 2 e 3 , que correspondem às mesorregiões da Borborema, Agreste e Zona da Mata, foram estatisticamente semelhantes em algumas características, como a exploração tipo leite, criação confinada, número de vacas em lactação, produtividade diária de leite e número de bovinos no rebanho; porém, tais circuitos foram estatisticamente diferentes do circuito produtor 1, que abrange a mesorregião do Sertão, em relação a essas características. Dessa maneira, sugere-se que tais semelhanças e diferenças sejam respeitadas quando do planejamento de políticas de desenvolvimento pecuário, bem como implementação de ações de controle de doenças. 


\section{Agradecimentos}

Ao Conselho Nacional de Desenvolvimento Científico e Tecnológico (CNPq) e à Coordenação de Aperfeiçoamento de Pessoal de Nível Superior (CAPES) pelo auxílio financeiro. Ao Serviço de Defesa Agropecuária do Estado da Paraíba, pela condução do trabalho de campo.

\section{Referências}

ALMEIDA, A. C. S.; FERREIRA, R. L. C.; SANTOS, M. V. F.; SILVA, J. A. A.; LIRA, M. A. Caracterização de produtores e propriedades rurais em três municípios do estado de Pernambuco. Revista Caatinga, Mossoró, v. 19, n. 4, p. 323-332, 2006.

ALVES, A. J. S. Caracterização epidemiológica da brucelose bovina no Estado da Bahia. 2008. Dissertação (Mestrado em Epidemiologia Experimental e Aplicada às Zoonoses) - Universidade de São Paulo, São Paulo.

AZEVEDO, S. S. Caracterização epidemiológica da brucelose bovina no Estado do Espírito Santo. 2006. Tese (Doutorado em Epidemiologia Experimental e Aplicada às Zoonoses) - Universidade de São Paulo, São Paulo.

BORBA, M. R. Caracterização epidemiológica da brucelose bovina no Estado do Maranhão. 2012. Tese (Doutorado em Epidemiologia Experimental e Aplicada às Zoonoses) - Universidade de São Paulo, São Paulo.

CARNEIRO, T. S.; ALVES, A. A.; AZEVÊDO, D. M. M. R.; BEZERRA, E. E. A.; CATALANO, D. Caracterização e eficiência produtiva de rebanhos bovinos leiteiros participantes do programa INFOLEITE no Baixo Parnaíba, Piauí. Revista Científica de Produção Animal, Teresina, v. 8, n. 2, p. 9-14, 2006.

CARVAlHO, M. V. B. M. A.; FERREIRA, R. L. C.; SANTOS, M. V. F.; DUBEUX JÚNIOR, J. C. B.; FREITAS, M. A. M.; ALMEIDA, O. C. Caracterização de propriedades rurais e identificação de espécies arbóreas e arbustivas ocorrentes em pastagens do Agreste de Pernambuco. Revista Científica de Produção Animal, Teresina, v. 3, n. 1, p. 38-54, 2001.

CEZAR, I. M.; QUEIROZ, H. P.; THIAGO, L. R. L. S.; CASSAlES, F. L. G.; COSTA, F. P. Sistemas de produção de gado de corte no Brasil: uma descrição com ênfase no regime alimentar e no abate. Campo Grande: Embrapa Gado de Corte, 2005. 40 p.

CONFEDERAÇÃO NACIONAL DE AGRICULTURA - CNA. Análise do PIB das cadeias produtivas de algodão, cana-de-açúcar, soja, pecuária de corte e de leite no Brasil: desenvolvimento metodológico e cálculo do PIB das cadeias produtivas do algodão, cana-de-açúcar, soja, pecuária de corte e de leite no Brasil. Brasília: CNA, 2012. Disponível em: <http://www.canaldoprodutor. com.br/sites/default/files/pib-cadeias-produtivas-web. pdf $>$. Acesso em: 05 maio 2013.

DIAS, R. A. Caracterização espacial da brucelose bovina no Estado de São Paulo. 2004. Tese (Doutorado em Epidemiologia Experimental e Aplicada às Zoonoses) - Universidade de São Paulo, São Paulo.

GOMES, A. T. Gerenciamento na atividade leiteira. Jornal da Produção de Leite - PDPL / RV, Viçosa, v. 12, n. 197, p. 254-261, 2005.

INSTITUTO BRASILEIRO DE GEOGRAFIA E ESTATÍSTICA - IBGE. Anuário estatístico do Brasil. Rio de Janeiro: IBGE, 2001. Disponível em: $<\mathrm{http}: / /$ biblioteca.ibge.gov.br/d detalhes.php?id=720>. Acesso em: 02 ago. 2013.

Censo Agropecuário 2006: Brasil, grandes regiões e unidades da federação. Rio de Janeiro: IBGE, 2009. Disponível em: <http://www.ibge.gov.br/home/ estatistica/economia/agropecuaria/censoagro/2006/ agropecuario.pdf $>$. Acesso em: 05 maio 2013.

Produção da Pecuária Municipal. Rio de Janeiro: IBGE, 2012. Disponível em: <http://www.ibge.gov.br/ home/estatistica/economia/ppm/2011/default.shtm>. Acesso em: 05 maio 2013.

INSTITUTO DE DESENVOLVIMENTO MUNICIPAL E ESTADUAL - IDEME. IDEME e IBGE publicam os resultados do PIB em 2009. João Pessoa: IDEME, 2011. Disponível em: <http://ideme.pb.gov.br/index.php/ objetivos-do-milenio/doc_details/1444-nota-tecnica-pibdos-municipios-2009.html $>$. Acesso em: 20 maio 2013.

LEITE, R. H. M.; LAGE, A. P.; JAIME, V. S.; MODENA, C. M. Perfil produtivo-sanitário de propriedades produtoras de bovinos do estado da Paraíba, Brasil, 2000. Ciência Animal Brasileira, Goiânia, v. 5, n. 4, p. 199209, 2004.

MIGUEL, L. A.; MIELITZ NETTO, C. G. A.; NABINGER, C.; SANGUINÉ, E.; WALQUIL, P. D.; SCHNEIDER, S. Caracterização socioeconômica e produtiva da bovinocultura de corte no estado do Rio Grande do Sul. Revista Estudo e Debate, Lajeado, v. 14, n. 2, p. 95-125, 2007.

MINISTÉRIO DA AGRICULTURA, PECUÁRIA E ABASTECIMENTO - MAPA. Exportação. Brasília: MAPA, 2013. Disponível em: <http://www.agricultura. gov.br/animal>. Acesso em: 05 maio 2013.

MONTEIRO, A. M.; TAMANINI, R.; SILVA, L. C. C.; MATTOS, M. R.; MAGNANI, D. F.; D’OVIDIO, L.; 
NERO, L. A.; BARROS, M. A. F.; PIRES, E. M. F.; PAQUEREAU, E. P. D.; BELOTI, V. Características da produção leiteira da região do agreste do estado de Pernambuco, Brasil. Semina: Ciências Agrárias, Londrina, v. 28, n. 4, p. 665-674, 2007.

MOURA, J. F. P.; PIMENTA FILHO, E. C.; GONZAGA NETO, S.; LEITE, S. V. F.; GUILHERMINO, M. M.; MENZES, M. P. C. Análise econômica da exploração de leite no cariri paraibano. Acta Scientiarum. Animal Sciences, Maringá, v. 32, n. 2, p. 225-231, 2010a.

MOURA, J. F. P.; PIMENTA FILHO, E. C.; GONZAGA NETO, S.; MENZES, M. P. C.; LEITE, S. V. F.; GUILHERMINO, M. M. Caracterização dos sistemas de produção de leite bovino no Cariri paraibano. Acta Scientiarum. Animal Sciences, Maringá, v. 32, n. 3, p. 293-298, 2010b.

NEVES, A. L. A.; PEREIRA, L. G. R.; SANTOS, R. D.; ARAÚJO, G. G. L.; CARNEIRO, A. V.; MORAES, S. A.; SPANIOL, C. M. O.; ARAGÃO, A. S. L. Caracterização dos produtores e dos sistemas de produção de leite no perímetro irrigado de Petrolina/PE. Revista Brasileira de Saúde e Produção Animal, Salvador, v. 12, n. 1, p. 209223, 2011.

PEREIRA, M. N. Conceitos para definição de sistemas de produção de leite no Brasil. Lavras: UFLA/Faepe, 2001. $167 \mathrm{p}$.

POLAQUINI, L. E. M.; SOUZA, J. G.; GEBARA, J. J. Transformações técnico-produtivas e comerciais na pecuária de corte brasileira a partir da década de 90 . Revista Brasileira de Zootecnia, Viçosa, MG, v. 35, n. 1, p. 321-327, 2006.
SIKUSAWA, S. Prevalência e caracterização epidemiológica da brucelose bovina no Estado de Santa Catarina. 2004. Dissertação (Mestrado em Epidemiologia Experimental e Aplicada às Zoonoses) - Universidade de São Paulo, São Paulo.

SILVA, R. A.; FERNANDES FILHO, S.; OLIVEIRA, A. V. B.; ARAÚJO, A. S.; SILVA, F. O.; PEREIRA, E. M. Caracterização do sistema de produção de leite do município de Paulista - PB. Agropecuária Científica no Semi-Árido, Patos, v. 6, n. 2, p. 31-46, 2010.

SILVEIRA, E. Z.; GROFF, A. M.; MARQUES, J. A.; BARBI, J. A. Caracterização da pecuária de corte na região de Tapejara - PR. Campo Digital, Campo Mourão, v. 1, n. 1, p. 48-53, 2006.

THRUSFIELD, M. Veterinary epidemiology. 2. ed. Cambridge: Blackwell Science, 1995. 479 p.

UNITED STATES DEPARTMENT OF AGRICULTURE

- USDA. Production, supply and distribution online - Dairy. Washington: USDA, 2011a. Disponível em: $<\mathrm{http} / / /$ www.fas.usda.gov/psdonline $>$. Acesso em: 20 maio 2013.

. Production, supply and distribution online Livestock. Washington: USDA, 2011b. Available at: $<\mathrm{http} / / / \mathrm{www}$.fas.usda.gov/psdonline $>$. Accessed at: 20 maio 2013.

VILLAR, K. S. Caracterização epidemiológica da brucelose bovina no Estado de Rondônia. 2008. Dissertação (Mestrado em Epidemiologia Experimental e Aplicada às Zoonoses) - Universidade de São Paulo, São Paulo.

ZAR, J. H. Biostatistical analysis. 4. ed. Upper Saddle River: Prentice Hall, 1999. 663 p. 
